\title{
Interview with Line Matthiessen
}

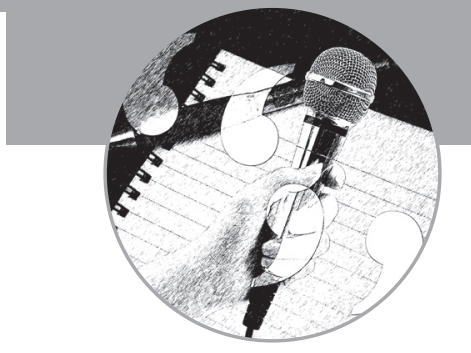

Line Matthiessen leads the Unit for Infectious Diseases and Public Health in the European Commission's Research and Innovation Directorate-General. Her unit promotes and supports EU research and innovation in the area of global health with emphasis on HIVIAIDS, malaria and tuberculosis, emerging epidemics, neglected infectious diseases and antimicrobial drug resistance, as well as activities in public health and health systems research. The Unit also supports the implementation of the European and Developing Countries Clinical Trials Partnership. Matthiessen was Head of Unit for coordination in the Directorate for Biotechnologies, Agriculture and Food Research from July 2007 to December 2010. She joined the European Commission in 1992. Matthiessen was trained as an MD at the University of Odense (Denmark) and received her PhD in Neurosciences from the University of Paris VI (France) in 1993. She spoke to Future Medicinal Chemistry regarding her career to date, how resistance has impacted neglected diseases and how the European Commission will tackle the issue of resistance in the coming years.

Interview conducted by Isaac Bruce, Managing Commissioning Editor.

Q How did you become involved in the field of drug resistance?

I have followed microbial drug resistance since my medical studies in Denmark back in the 1980s where we had already learned the importance of the prudent use of antibiotics. I became involved specifically in antimicrobial drug resistance (AMR) in 2011 when I was appointed Head of Unit for Infectious Diseases and Public Health in the European Commission's Research and Innovation Directorate-General.

Can you update us on some of the Unit of Infectious Diseases and Public Health's current work with regard to combating resistance to antimicrobial drugs?

Antimicrobial resistance is a growing health problem that requires swift and determined action. Together with our Health and Consumers Directorate-General we are currently implementing the Commission's Action Plan against AMR from November 2011. A greater research and innovation effort is an important element of this plan - to develop new tools to combat AMR, provide the best possible care for patients and further develop a science-based health policy.

We work mainly along three lines of action. The first is collaborative research and innovation. We get leading players from Europe and across the globe to team up and work together within EU-funded projects to develop new and innovative solutions for the AMR challenge. Our projects have a variety of complementary aims, for example, to improve our understanding of the interactions between microbes and humans, to identify compounds that might lead to new drugs, to develop new diagnostic tests and approaches for controlling and monitoring the spread of AMR. Altogether they address the full scope of AMR, including bacterial, viral, protozoan and fungal infections. Furthermore, the optimum use of currently available antibiotics is being investigated in clinical trials funded by the EU.

Our second action line is the Innovative Medicines Initiative (IMI), Europe's largest publicprivate partnership to develop better and safer medicines for patients. Within this partnership, we have developed, together with the European Federation of Pharmaceutical Industries and Associations, the $€ 350$ million research program 'New Drugs for Bad Bugs' to bring new antibiotics to patients. Two major projects worth $€ 224$ million just kicked off under this program earlier this year.

Third, we support the EU Member States in their efforts to work together in a more coordinated and strategic way with the aim to create synergies between national research funding programs on AMR. The scientific advisory board of this so-called 'Joint Programming Initiative' [1] is currently developing the strategic research agenda that will serve as a basis for joint research calls between the participating European Member States.

We are doing a lot of important work on AMR and you can expect that this effort will also continue under the EU's 2014-2020 Research And Innovation Framework Program 'Horizon 2020', for which we are currently defining the priorities.

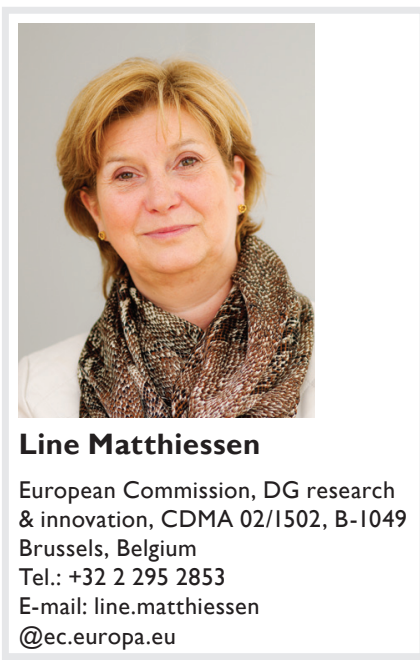

FUTURE
SCIENCE FSS 


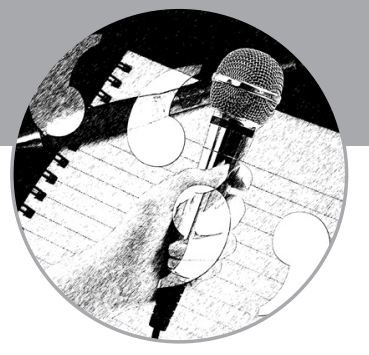

Q The Unit of Infectious Diseases and Public Health has great involvement with neglected diseases. Resistance to drugs to treat these diseases could have a devastating effect in the developing world: what new strategies are emerging to help prevent this?

One of the most popular strategies for controlling some of the neglected infectious diseases (NIDs), for example, river blindness, has been so-called mass drug administration, where large population groups have indiscriminately been given drugs. In other situations, such a strategy has led to the development of drug resistance. This is certainly also a risk for NIDs, where the combination of drug resistance and resource-poor settings would be devastating. One of the key strategies against drug resistance for NIDs is, therefore, simply to develop new drugs and, thereby, get an alternative to apply if/when drug resistance appears. So far, we have invested more than $€ 75$ million into NID research under the current 'Framework Programme', mostly to develop new vaccines, treatments and diagnostics for NIDs. Many NIDs can be treated with existing drugs, but often there is only a single drug available. We are also aware of the growing problem of drug resistance among these diseases. Unfortunately, when resistance emerges, there are simply no 'second-line' drugs. This is, for instance, the case with many of the helminthic diseases such as river blindness and schistosomiasis. In such a situation drug resistance would have devastating effects for the many millions of people in poor countries affected by these diseases. The other strategy would be to support the introduction of effective surveillance systems in developing countries, which would make it possible to rapidly detect emerging diseases, as well as emerging drug resistance. This is, however, a long-term goal, as it will require a number of developments, in terms of technologies that are suitable to be used for surveillance and detection in resource-poor settings. We are also funding a number of projects addressing these technical gaps [2].

\section{Q To date, what have been the Unit of Infectious Diseases and Public Health's biggest} successes in relation to combating resistance?

I can give you a few examples from our collaborative research projects: the GRACE project, which involved thousands of patients in a placebo-controlled trial, could demonstrate that prescribing antibiotics for uncomplicated lower respiratory tract infections does not improve the clinical outcome. Another project, Actinogen, has led to the discovery and patenting of an entirely novel 'lead antibiotic' that can be taken up in drug development. Other projects have identified potent biofilm-inhibiting compounds, developed diagnostic tools to detect (resistant) bacterial strains, focused on the spread of resistant infections, or studied a range of determinants, such as bacterial fitness, virulence and survival. Finally, the MOSAR project has linked epidemiological data to clinical and microbiological information and analyzed and characterized more than 10,000 bacterial isolates. These works have greatly improved our understanding of the types of bacteria causing infections in healthcare settings.

Q Do you think big pharmaceutical companies are doing their fair share to help tackle drug resistance and neglected diseases?

It is true that the number of large pharmaceutical companies that remain in the antibiotic R\&D field has gone down drastically from 19 in 1990, to only four in 2012. There are several reasons for the lack of industrial investment in new antibiotics. Developing new, effective and safe antibiotics is more and more scientifically difficult and costly. In addition, restrictions on the use of antibiotics and their underpricing compared with other therapeutic classes deter investment. If this situation were to continue we would risk ending up in a situation where many bacterial infections can no longer be treated, which also jeopardizes advanced surgery, chemotherapy and care of preterm babies. It is, therefore, crucial that public and private partners collaborate to find solutions for this serious health threat. Our IMI partnership with its 'New Drugs for Bad Bugs' program is a very timely and powerful initiative in this context, and we hope that it will help bring much needed new antibiotics to patients. We are also working with industry and other stakeholders to develop a new business model for antibiotics as we are faced with the paradox that we want to stimulate the development of new antibiotics, but at the same time we want to restrict the use of these drugs.

Q Do you think that innovation in regards to combating drug resistance is hindered by a lack of open collaboration among companies and the sharing of resources and research? Faced with the dwindling number of companies that are active in the area of antimicrobial development and the fact that scientifically it is more and more difficult to develop these drugs, researchers 
in companies and academia are convinced that sharing of resources is crucial to make progress in this area. The new IMI project COMBACTE launched earlier this year is pioneering a new collaborative approach which will hopefully result in a new model for the clinical research and development of antibiotics. An important element in these projects is to increase the efficiency of antibiotic $\mathrm{R} \& \mathrm{D}$ through knowledge and data sharing, to avoid duplication and learn from successes and failures. This model will see academic investigators working hand-in-hand with industry scientists to combine knowledge and expertise, thereby increasing the probability of developing effective new medicines and addressing the public health threat of AMR. A key outcome of the project will be a pan-European clinical trial network capable of recruiting patients and conducting multinational trials at all stages of drug development.

What role do you see for small and medium enterprises?

We launched, last year in July, a specific funding round with emphasis on small and medium enterprises (SMEs) working in the area of AMR. We invited SMEs to submit project proposals aiming at developing new drugs, vaccines or other medical alternatives against infections that have developed or are at the risk of developing drug resistance. At least half of the EU funding should go to SMEs that take part in projects with three-five partners from at least three different European countries. We are currently finalizing the funding arrangements of the seven best project proposals of this call, which involve 17 SMEs from France, Germany, Italy, Belgium, Switzerland, Denmark, Ireland, UK and Spain. These projects will aim to develop new drugs or vaccines against bacterial, fungal and viral infection. The big response - we had 78 funding applications initially - clearly demonstrates the interest and degree of SME activity in this area. These companies hold some of the keys to innovation and progress in the field.

How do you anticipate the field will progress in the next 5-10 years?

It is important to realize that this is not a national or European problem. It is a global problem that requires that we work more closely in co-operation with other world regions and our partners from across the globe. We have exchanges with our colleagues form the USA and other countries, but we also need to start a dialog with countries, such as China or India, where the vast majority of people are now living and where antibiotics are used excessively. Pathogens are fast-moving global players, thus, we need to coordinate our efforts and exchange information better to be able to react rapidly and eliminate drug resistance before it spreads over a large area and becomes impossible to contain.

What words of advice do you have for a young scientist envisioning a career in drug resistance research?

I wish to strongly encourage them to join the combat against drug resistance. We urgently need new innovative ideas from bright young scientists.

\section{Disclaimer}

The views expressed in this interview are the sole responsibility of the interviewee and do not necessarily reflect the views of the European Commission or of Future Science Ltd. Neither the European Commission, nor any person acting on behalf of the Commission, is responsible for the use that might be made of the information contained therein.

\section{Financial \& competing interests disclosure}

The interviewee has no relevant affiliations or financial involvement with any organization or entity with a financial interest in or financial conflict with the subject matter or materials discussed in the manuscript. This includes employment, consultancies, honoraria, stock ownership or options, expert testimony, grants or patents received or pending, or royalties.

No writing assistance was utilized in the production of this manuscript.

\section{Websites}

1 Joint programming initiative on antimicrobial resistance.

www.jpiamr.eu
2 EC Research in neglected infectious diseases. http://ec.europa.eu/research/health/infectiousdiseases/neglected-diseases/index_en.html 